\title{
Comment enrichir les outils professionnels à l'aide de concepts et outils issus de la recherche dans les didactiques disciplinaires?
}

How to link professional tools to research tools in comparative educational research? An example in French and Physics teaching

\section{Anne Monnier et Laura Weiss}

\section{OpenEdition \\ Journals}

Édition électronique

URL : https://journals.openedition.org/educationdidactique/892

DOI : 10.4000 /educationdidactique.892

ISBN : 978-2-7535-1625-0

ISSN : 2111-4838

Éditeur

Presses universitaires de Rennes

Édition imprimée

Date de publication : 15 décembre 2010

Pagination : $97-118$

ISBN : 978-2-7535-1301-3

ISSN : 1956-3485

Référence électronique

Anne Monnier et Laura Weiss, « Comment enrichir les outils professionnels à l'aide de concepts et outils issus de la recherche dans les didactiques disciplinaires ? », Éducation et didactique [En ligne], 4-3 | Décembre 2010, mis en ligne le 10 décembre 2012, consulté le 22 juillet 2022. URL : http:// journals.openedition.org/educationdidactique/892 ; DOI : https://doi.org/10.4000/ educationdidactique. 892 


\title{
COMMENT ENRICHIR LES OUTILS PROFESSIONNELS À L'AIDE DE CONCEPTS ET OUTILS ISSUS DE LA RECHERCHE DANS LES DIDACTIQUES DISCIPLINAIRES?
}

\author{
RÉFLEXIONS AUTOUR DE L'ENSEIGNEMENT DE LA \\ GRAMMAIRE ET DE LA MASSE VOLUMIQUE EN FORMATION \\ INITIALE DES ENSEIGNANTS DU SECONDAIRE.
}

\author{
Anne Monnier (Equipe GRAFE, IUFE, Université de Genève) \\ Laura Weiss (IUFE, Université de Genève)
}

\begin{abstract}
Résumé :Cet article fait état d'une recherche portant sur un dispositif de formation en didactique disciplinaire qui tente d'articuler un outil professionnel de planification de l'enseignement, le canevas de leçon, à un outil issu de la recherche en didactique du français, le synopsis. L'analyse comparative d'un ensemble de données recueillies dans le cadre d'une expérimentation menée à l'IUFE de Genève avec des enseignants du secondaire à mi-temps en emploi, d'une part en français à propos de l'enseignement de la subordonnée relative, d'autre part en sciences physiques à propos de l'enseignement de la masse volumique, aboutit à deux constats. A un premier niveau, si l'articulation entre outils professionnels et outils issus de la recherche se révèle difficile, par le fait qu'elle implique la mise en place de dispositifs relativement complexes, elle est prometteuse dans le cadre d'une formation désormais universitarisée. A un second niveau, l'analyse comparative d'un dispositif de formation sur le même outil dans deux didactiques spécifiques ouvre des pistes intéressantes du point de vue de la recherche en didactique comparée.
\end{abstract}

Mots-clés : acceptabilité, utilisabilité, utilité, artefact, canevas, didactique du français, didactique des sciences, didactique professionnelle, didactique comparée, enseignants du secondaire, formation initiale, grammaire, instrument, masse volumique, outi, pratique réflexive, synopsis.

Anne Monnier \& Laura Weiss

\section{Introduction}

Cette contribution s'inscrit dans le cadre de la phase de transition entre l'ancien institut de formation des enseignants du secondaire à Genève (IFMES) et la mise en place du nouvel institut universitaire de formation des enseignants du secondaire (IUFE). En tant que formatrices-chercheuses qui participons à la définition, à la production, et à la diffusion des savoirs de didactique en français et en sciences, une de nos préoccupations est l'élaboration de contenus de formation en didactique en lien avec trois contraintes fortes. Premièrement, ces contenus doivent viser la maîtrise de savoirs professionnels, en vue de l'exercice de la profession selon les normes officiellement fixées par la CDIP ${ }^{1}$ et par la LIP $^{2}$. Deuxièmement, ces contenus doivent être adaptés au profil spécifique des étudiants de cette phase de transition: porteurs d'un master, voire d'un doctorat universitaire dans leur(s) discipline(s) de formation, la plupart d'entre eux arrivent à l'IUFE avec un bagage académique important, mais également, pour une partie d'entre eux, avec une expérience professionnelle hors enseignement. Troisièmement, il s'agit de prendre en compte, pour mieux le déjouer, le préjugé, encore extrêmement répandu au niveau des professionnels, selon lequel une formation pédagogique est inutile pour enseigner au niveau secondaire. Les étudiants sont en effet fortement influencés par les opinions de leurs collègues expérimentés, étant engagés, dans cette phase de transition, dès leur première année de formation, à mi-temps dans un établissement scolaire secondaire obligatoire (élèves de 13 à 15 ans) ou post-obligatoire (élèves de 16 à 20 ans).

À partir de ces prémisses, quels savoirs privilégier dans les ateliers de didactique qui sont le lieu privilégié pour articuler théorie et pratique? Ne convient-il pas d'y travailler d'une part les savoirs présents dans les plans d'études du secondaire, en particulier ceux qui ne sont pas étudiés à l'Université, d'autre part un certain nombre de savoirs spéci- 
fiques au processus « enseigner »? Pour ancrer ces savoirs dans un fondement théorique, n'y aurait-il pas aussi un intérêt pour les formateurs à s'inspirer des concepts et outils produits par la recherche en didactique?

Pour tenter de répondre à ces questions, nous procédons de la façon suivante. Après avoir défini l'inscription théorique de cette recherche et affiné la problématique, nous présentons et analysons le dispositif de formation expérimenté autour du canevas de leçon, en lien avec un outil issu de la recherche, le synopsis. Pour ce faire, nous définissons les concepts et outils proposés, indiquons la méthodologie choisie et présentons les résultats de notre analyse. Nous concluons ce texte par une réflexion sur les liens entre formation et recherche que le dispositif analysé questionne et sur la possibilité et la pertinence de transposer ces concepts et outils à d'autres didactiques disciplinaires dans le cadre de la formation des enseignants.

\section{Cadres théoriques}

\section{Inscription de la recherche en didactiques disci- plinaires et en didactique comparée}

Notre étude se situe d'abord en didactique du français et en didactique des sciences, puisqu'elle garde une focalisation forte sur l'enseignement d'un objet de savoir disciplinaire précis - la grammaire pour la première, la masse volumique pour la seconde, ensuite sur certains concepts et outils propres à ces didactiques.

Notre questionnement rejoint également à un double titre les préoccupations de la didactique comparée, telle que définie par Mercier, SchubauerLeoni et Sensevy (2002) et Leutenegger (2004). En effet, il porte sur les possibilités de faire émerger et/ ou de conférer un aspect générique aux concepts et outils des didactiques spécifiques grâce à l'interaction dialectique de concepts issus de différents champs de recherche. Sensibles cependant aux dérives possibles de la migration des concepts entre didactiques voisines, nous faisons nôtre la proposition de Johsua de « réserver le terme didactique à toutes les situations d'étude que l'on peut analyser ou éclaircir en suivant prioritairement le fil du rapport au savoir » (Johsua,
2002, p. 19). Autrement dit, la migration de concepts d'une didactique à l'autre ne se justifie que si le concept en question permet une meilleure compréhension du déploiement d'un objet de savoir précis dans le contexte de la classe. Qui plus est, en proposant en parallèle à des étudiants de physique et à des étudiants de français un dispositif de formation portant sur le même outil professionnel, nous cherchons à voir dans quelle mesure les spécificités de ces deux disciplines ont un impact sur l'outil proposé et l'infléchissent $a$ priori dans une direction ou dans une autre. Ces disciplines sont en effet très différentes, non seulement du point de vue de leur matrice disciplinaire et de leur logique interne, mais également du point de vue de leur statut dans le système éducatif genevois - si le français est enseigné depuis l'école primaire avec une dotation horaire importante, la physique n'intervient qu'à partir de la fin de l'école obligatoire (élèves de 14-15 ans), à raison d'une seule fois par semaine.

En outre, nous nous appuyons sur les composantes du triplet développé en didactique comparée par Schubauer-Leoni et Leutenegger (2002) pour l'analyse de leçons: la chronogenèse qui se concrétise dans l'avancement du temps didactique par rapport au temps d'apprentissage, la topogenèse qui décrit les rôles des différents acteurs du triangle didactique et la mésogenèse qui se traduit dans les tâches proposées en fonction des objectifs poursuivis. Ce triplet, développé au départ pour la didactique des mathématiques, comporte un aspect générique qui lui permet d'être également opérationnel pour l'analyse de leçons en français et en physique.

\section{Inscription de la recherche en didactique profes- sionnelle et recours à des concepts d'ergonomie}

À un deuxième niveau, nous inscrivons cette recherche en didactique professionnelle, car nous nous intéressons à un dispositif de formation professionnelle qui s'appuie sur l'analyse de «l'activité du praticien » pour reprendre l'expression de Pastré (2004). Dans ce cadre, il s'agit de voir ici dans quelle mesure un outil professionnel de planification de l'enseignement se révèle utile pour des enseignants en formation. Riff et Durand (1993), dans leur revue de littérature consacrée à l'analyse du travail de l'enseignant, aussi bien en classe que privé, traitent de l'activité de planification et mettent en évidence les 
différences entre enseignants novices et enseignants experts. Selon eux, la planification de l'enseignement peut être définie comme « une activité de sélection dans un répertoire de routines et $[. .$.$] une réflexion$ relative à leur mise en ouvre » (p. 100). Ainsi, l'activité anticipatrice de planification serait toujours présente chez les experts, mais, dans la mesure où elle est liée à des routines, elle s'appuie essentiellement sur ce qu'ils appellent « des images mentales anticipatrices $»$ (p. 88), et ne prend pas forcément la forme de traces écrites. Au contraire, une telle organisation n'existe pas en mémoire chez le débutant qui ne possède que peu ou pas de routines, ce qui expliquerait pourquoi cette activité anticipatrice est difficile chez les novices. D'où l'importance de proposer en formation initiale des outils de planification, qui sont d'abord des outils de formation et qui peuvent acquérir le statut d'outils professionnels. Qu'entendons-nous cependant par « outil » et plus précisément « outil professionnel »?

Sans reprendre totalement la théorie de l'instrumentation de Rabardel (1995), nous reprenons sa définition de l'artefact comme partie neutre et universelle de l'instrument, indépendante de l'usage qu'en fait l'utilisateur: «Chaque artefact a été conçu pour produire une classe d'effets, et sa mise en œuvre, dans des conditions prévues par les concepteurs, permet d'actualiser ces efforts. Autrement dit, à chaque artefact correspondent des possibilités de transformations des objets de l'activité, qui ont été anticipées, délibérément recherchées et qui sont susceptibles de s'actualiser dans l'usage. En ce sens, l'artefact (qu'il soit matériel ou non) concrétise une solution à un problème ou à une classe de problèmes socialement posés » (p. 59). Linstrument, au contraire, est l'artefact en situation, employé pour un usage particulier et précis: "Nous utiliserons le terme d'instrument pour désigner l'artefact en situation, inscrit dans un usage, dans un rapport instrumental à l'action du sujet, en tant que moyen de celle-ci. » (p. 59). Dans notre situation, l'outil de planification proposé a un statut d'artefact conçu par les formateurs que les étudiants peuvent transformer, dans leur pratique, en un instrument de préparation de cours. En cela, il peut être considéré comme un outil professionnel, c'est-à-dire utile dans leur pratique professionnelle, au même titre que le tableau noir en classe (Nonnon, 2000) ou le cahier de plans dans le cas des sapeurs pompiers (Perez \& Rogalski, 2001).
Tricot et al. (2003) proposent trois dimensions pour l'évaluation de l'utilisation des Environnements Informatiques pour l'Apprentissage Humain (EIAH), qui nous semblent pouvoir être transposées de façon pertinente à un artefact de planification de l'enseignement: l'utilité ou l'efficacité d'un outil; l'utilisabilité, qui recouvre la maniabilité de ce dernier, et l'acceptabilité, qui correspond à la valeur des attitudes et opinions plus ou moins positives à propos d'un outil. Selon eux en effet, " de très bons outils, très bien promus par leurs concepteurs, voire remarquablement étayés d'un point de vue didactique, se révèlent inutilisables. Par ailleurs, des outils pourtant faciles à utiliser ne parviennent pas à entrer dans les pratiques scolaires ou de formation professionnelle. Certains enseignants n'ont pas le temps, les compétences ou l'envie de les utiliser [...]. Un bon EIAH est donc non seulement utile à l'apprentissage visé, mais encore utilisable et acceptable» (p. 391-392). À partir de là, ils établissent différents critères pour évaluer empiriquement ces trois dimensions qui peuvent être reprises en partie pour l'évaluation de notre double dispositif de formation: pour la dimension " utilité », les critères « précision et présentation des objectifs, adéquation contenus/objectifs, précision du scénario didactique, adéquation scénario/objectifs/contenus »; pour la dimension « utilisabilité », les critères « efficience, charge de travail, signifiance des codes et dénominations »; enfin, pour la dimension « acceptabilité », les critères « adéquation aux attentes et aux caractéristiques des apprenants ».

\section{Problématique}

Les ateliers des didactiques disciplinaires sont le lieu pour étudier aussi bien des savoirs à enseigner que des savoirs pour enseigner, les premiers pouvant être définis comme les objets du travail enseignant, les seconds constituant les outils du travail enseignant (Hofstetter \& Schneuwly, 2009). Si l'importance et le statut des savoirs à enseigner déterminés par les curriculums scolaires des disciplines ne sont pas remis en cause par les futurs enseignants du secondaire, il n'en va pas de même pour les seconds qui souvent ne sont pas considérés comme des savoirs de la formation. En effet, les enseignants du secondaire ont tendance à considérer qu'il s'agit d'outils ne pouvant s'acquérir que sur le terrain grâce aux conseils avisés des collègues expérimentés. Comment 
faire alors pour que les futurs enseignants acceptent de travailler en formation sur ces outils, en particulier ceux de planification de l'enseignement? En reprenant la conceptualisation de Rabardel (1995), quels artefacts proposer que les étudiants jugent utilisables et utiles et qu'ils instrumentalisent en fonction de leurs besoins pour la préparation de leurs leçons? En articulant certains outils professionnels à des concepts et à des outils issus de la recherche en didactique, ne leur confère-t-on pas une facette conceptuelle qui contraint à la réflexion sur les savoirs convoqués?

Pour tenter de répondre à ces questions, nous avons élaboré un dispositif combinant savoirs à enseigner et savoirs pour enseigner, qui a été expérimenté en parallèle dans les deux disciplines.

\section{Description du dispositif expérimenté}

Les ateliers de didactique traitant de la planification, dans le cadre desquels s'est déroulée cette recherche, s'étendent environ sur un semestre en suivant une logique allant du plus large au plus pointu. Il s'agit d'aborder la planification à long terme avec les questions de curriculum et de plans d'études, à moyen terme avec tout un travail autour des séquences didactiques, et à court terme avec l'analyse a priori d'une tâche et le canevas de leçon. À l'intérieur de cette thématique générale, un certain nombre de savoirs à enseigner sont abordés, en lien avec les plans d'études de l'enseignement secondaire. Il faut en effet se souvenir que certains contenus ne font pas partie des curriculums universitaires académiques, parce qu'ils sont considérés comme des prérequis, acquis par les étudiants lors de leur scolarité secondaire. De plus, dans les études universitaires - en cohérence avec leur objectif qui n'est pas de former des enseignants de la discipline - il n'est pas fait référence aux difficultés d'enseignement de certaines notions et l'histoire des savoirs y est rarement abordée, alors qu'elle peut être une clé explicative des difficultés d'apprentissage. Cependant, il est impossible dans la formation didactique des enseignants d'aborder de façon systématique toutes les notions enseignées. C'est pourquoi, dans notre dispositif, les savoirs à enseigner sont choisis en fonction des difficultés qu'ils recèlent pour les élèves ou de certaines lacunes des étudiants. Notre pari est en effet le suivant: dans la mesure où il n'est pas possible de préparer les étudiants à faire face à toutes les situations auxquelles ils seront confrontés dans leur classe et de travailler avec eux tous les objets de savoirs qu'ils seront amenés à enseigner, il convient de leur proposer des situations qui, sans constituer des modèles à imiter, soient suffisamment prototypiques pour permettre une transposition des compétences exercées, aussi bien dans d'autres situations de classe qu'en lien avec l'enseignement d'autres objets de savoir du même domaine.

Nous nous référons dans cet article, d'une part aux ateliers qui concernaient le canevas de leçon, d'autre part au transfert personnel sur le terrain par les étudiants des savoirs abordés lors de ces ateliers. Les séances avec les étudiants de français ne se sont cependant pas déroulées exactement de la même manière que celles destinées aux étudiants de physique. Dans les deux ateliers, l'outil canevas a été mis en relation avec des concepts didactiques spécifiques aux deux disciplines et utiles pour la planification de l'enseignement, comme par exemple le concept de séquence didactique en français ou celui de représentations initiales et d'obstacle en physique. Toutefois, si les premiers ont travaillé le canevas en articulation avec un outil issu de la recherche en didactique du français, le synopsis, les étudiants de physique ont travaillé le canevas sans lien avec l'outil en question, jouant d'une certaine manière le rôle de groupe témoin. Qui plus est, si les étudiants de français n'ont dû rédiger un canevas que pour une seule observation de leçon, en ayant de plus la liberté de transformer le modèle proposé en atelier selon leurs penchants et besoins, les étudiants de physique ont dû rédiger un canevas pour chaque observation de leçon par la formatrice, selon le modèle standard proposé en atelier. La démarche suivie par les formatrices pour l'observation et l'analyse des leçons des étudiants a été la même dans les deux groupes: élaboration d'un descriptif de la leçon observée, entretien avec l'étudiant, puis rédaction d'un compte rendu d'observation et d'analyse destiné à l'étudiant.

\section{Choix des savoirs pour enseigner}

Le canevas est un outil professionnel utilisé dans l'enseignement, aussi bien dans les petits degrés scolaires que dans les degrés supérieurs, pour la planification à court, moyen et long terme (Rey, 
1998; Clerc, 1995; Prégent, 1990, Sénore \& Ubaldi, 2006). Bien que n'ayant pas d'appellation univoque, ni de forme ou de contenu standard, le canevas de leçon, tel qu'on en trouve des modèles dans la littérature, comporte habituellement les rubriques suivantes: objectif(s) de la leçon, prérequis, insertion de la leçon dans la séquence, timing, matériel, formes sociales de travail, tâches de l'enseignant, tâches des élèves, suites (devoirs pour les élèves, travail à poursuivre par l'enseignant). Dans notre dispositif, nous avons mis l'accent sur le «canevas de leçon » correspondant à la planification d'une leçon unique, et non d'une séquence d'enseignement, pour la raison suivante. Comme les plans d'études d'une discipline sont définis par année scolaire, chaque enseignant doit organiser, pour chacune de ses classes, son enseignement en prévoyant un certain nombre de séquences traitant des thèmes prévus, généralement d'une durée de quelques semaines. Néanmoins, par opposition à l'enseignement primaire, où l'enseignant peut gérer le temps scolaire comme il l'entend, l'enseignement secondaire genevois est structuré temporellement de façon rigide en plages de 45 minutes. Un enseignant, en particulier s'il est débutant, ne peut faire abstraction de l'unité de temps dans laquelle il doit inscrire quotidiennement son enseignement.

Dans le dispositif avec les enseignants de français, le canevas a été articulé au synopsis et aux concepts sous-jacents à cet outil. Le synopsis est un outil méthodologique qui a été développé en didactique du français à la FPSE de Genève dans le cadre d'un projet de recherche visant « la description des pratiques scolaires effectives et la compréhension de la morphogenèse de l'objet enseigné » (GRAFE, 2005 , p. 2), pour condenser la grande masse de données d'une séquence d'enseignement en une unité appréhendable. Dans la version remaniée de cet outil (Monnier \& Weiss, 2009), il s'agit de découper, non plus une séquence filmée dans sa globalité, mais une partie de la séquence, une leçon ou un événement remarquable de celle-ci, en différentes instances en fonction de l'objet enseigné, de l'activité finalisée proposée aux élèves et de l'environnement mis en place pour l'activité selon une structure en niveaux et colonnes (voir annexe). Ce synopsis, élaboré a posteriori sur une leçon filmée pour décrire et analyser comment le savoir se déploie dans l'interaction enseignant-élèves, présente plusieurs similitudes structurelles avec le canevas de leçon: les indications tempo- relles, les formes sociales de travail, le matériel, les tâches et activités des élèves et de l'enseignant. C'est la raison pour laquelle il nous est apparu que l'élaboration d'un synopsis sur une leçon filmée d'un enseignant expérimenté peut permettre aux étudiants de mieux se représenter une leçon à partir des canevas possibles construits a priori pour un contenu donné.

Dans le dispositif avec les enseignants de physique, le canevas a été articulé à plusieurs concepts de la didactique des sciences. Il s'agit d'abord du concept d'obstacle (Astolfi \& Peterfalvi, 1993; Astolfi, 1997; Astolfi \& Develay, 2002), rendu plus opérationnel par la définition d'objectif-obstacle (Martinand, 1986, 1996) qui consiste à « coupler ces deux points de vue, ce qui les renouvelle partiellement. $\mathrm{Au}$ lieu de définir d'une part les objectifs sur la seule base apriorique de l'analyse de la matière, et d'autre part les obstacles psychologiques et épistémologiques sur la base de l'activité du sujet, il s'agit d'utiliser la caractérisation des obstacles comme un mode de sélection des objectifs » (Astolfi \& Develay, 2002, p. 56). Il s'agit ensuite du concept de phases didactiques qui, se référant initialement aux phases des situations didactiques de Brousseau (1998), ont été reprises par Briand et Chevalier (1995), et adaptées à la physique par Robardet et Guillaud (1997). Ces phases qui déterminent différents moments de l'enseignement-apprentissage permettent de travailler avec les enseignants en formation sur la structuration des leçons et les rôles respectifs de l'enseignant et des élèves.

\section{Choix des savoirs à enseigner}

La question s'est posée de savoir s'il était préférable de se limiter à ne travailler qu'un seul objet de savoir respectivement en français et en physique ou au contraire plusieurs à l'intérieur du même dispositif. Il nous a semblé plus riche de partir d'un sujet précis, laissant les étudiants utiliser l'outil ultérieurement, dans le cadre de leurs leçons, sur des objets de savoir différents. Ainsi, le choix du savoir à enseigner pour aborder le canevas en français s'est porté sur la subordonnée relative pour trois raisons. Ce sujet est présent dans les plans d'études du secondaire obligatoire (13 à 15 ans) et post-obligatoire (16 à 20 ans). Il comporte ensuite un certain nombre d'obstacles épistémiques pour les élèves, tout en étant particulièrement fécond pour développer des connaissances 
concernant certains mécanismes de la langue, mais également des compétences en production écrite, sans compter les possibilités qu'il ouvre du côté du développement de l'abstraction (Legrand, 1994). Enfin, les ateliers étant des lieux privilégiés pour des débats relatifs aux savoirs scolaires, il permet de revenir avec les étudiants sur les débats concernant l'enseignement de la grammaire, et de réfléchir à des questions comme: qu'est-ce que la grammaire? quelles en sont les finalités? existe-t-il une grammaire au-delà de la phrase? comment subordonner les activités de "structuration » aux activités d' " expression », comme le demandent les plans d'études actuels de français? Troisièmement, les profondes rénovations de la grammaire depuis les années 1980, et la parution entre autres de l'ouvrage Maîtrise du français (Besson, Genoud, Lipp, \& Nussbaum, 1979) ont entraîné, au niveau de l'enseignement de cette sous-discipline, un certain nombre de difficultés rappelées par De Pietro (2009): d'un côté il s'agit désormais « de subordonner la structuration à la communication, de rompre aussi avec une certaine tradition grammaticale $[\ldots]$; de l'autre côté, en lien avec les développements spectaculaires de la linguistique, les objectifs assignés au travail de structuration apparaissaient plutôt ambitieux » (p. 17). Il semble donc important de travailler ces difficultés avec les étudiants dans le cadre de la formation. À cela s'ajoute une raison technique non négligeable: le GRAFE a filmé treize séquences sur ce sujet en Suisse romande qui ont fait l'objet d'une analyse didactique approfondie (Schneuwly et al., 2009); nous avions donc un certain nombre de vidéos à disposition sur l'enseignement de ce sujet en $8^{\mathrm{e}}$ année.

En physique aussi, le savoir à enseigner a été choisi à partir d'un certain nombre de contraintes: le public d'étudiants enseignant aussi bien au secondaire obligatoire qu'au secondaire post-obligatoire imposait un thème présent dans tous les plans d'études du secondaire: la masse volumique. Le premier intérêt de ce sujet est qu'il présente une facette théorique, la modélisation algébrique à l'aide de l'expression $\rho=m / V$ et une facette empirique qui peut faire l'objet de différentes expériences réalisées par l'enseignant ou par les élèves, assez simples du point de vue du matériel requis et des manipulations nécessaires. Cette caractéristique permet aussi de réfléchir à la place du travail expérimental dans l'enseignement scientifique. Une deuxième raison de ce choix est qu'à l'instar d'autres grandeurs physiques intensives et parce qu'il s'agit d'un rapport de grandeurs, il comporte plusieurs obstacles épistémologiques. Un troisième argument en faveur de la masse volumique est le fait que sa mesure expérimentale implique d'aborder la notion de mesure et de son imprécision. Enfin, la masse volumique étant un prérequis pour d'autres notions, il s'agit d'inviter les enseignants à penser son enseignement pour qu'il constitue une base solide pour la suite.

Le schéma ci-dessous résume le projet: canevas et synopsis sont représentés par des flèches courbes, puisque ce sont des outils s'appliquant aux leçons en jeu, qu'elles soient réelles - celle donnée par l'enseignant filmé, représentée par des contours continus - ou potentielles - celles projetées à travers l'élaboration de leurs canevas, notées en pointillées. Quant aux objets de savoir, ceux abordés en formation sont étiquetés par le chiffre 1 (la subordonnée relative) ou l'(la masse volumique), alors que ceux choisis par les étudiants lors des leçons observées par la formatrice sont les « objet de savoir $\mathrm{x} »$ ou « objet de savoir x'», dans la mesure où il n'y a pas nécessairement de correspondance entre les premiers détaillés ci-dessus et les seconds. 


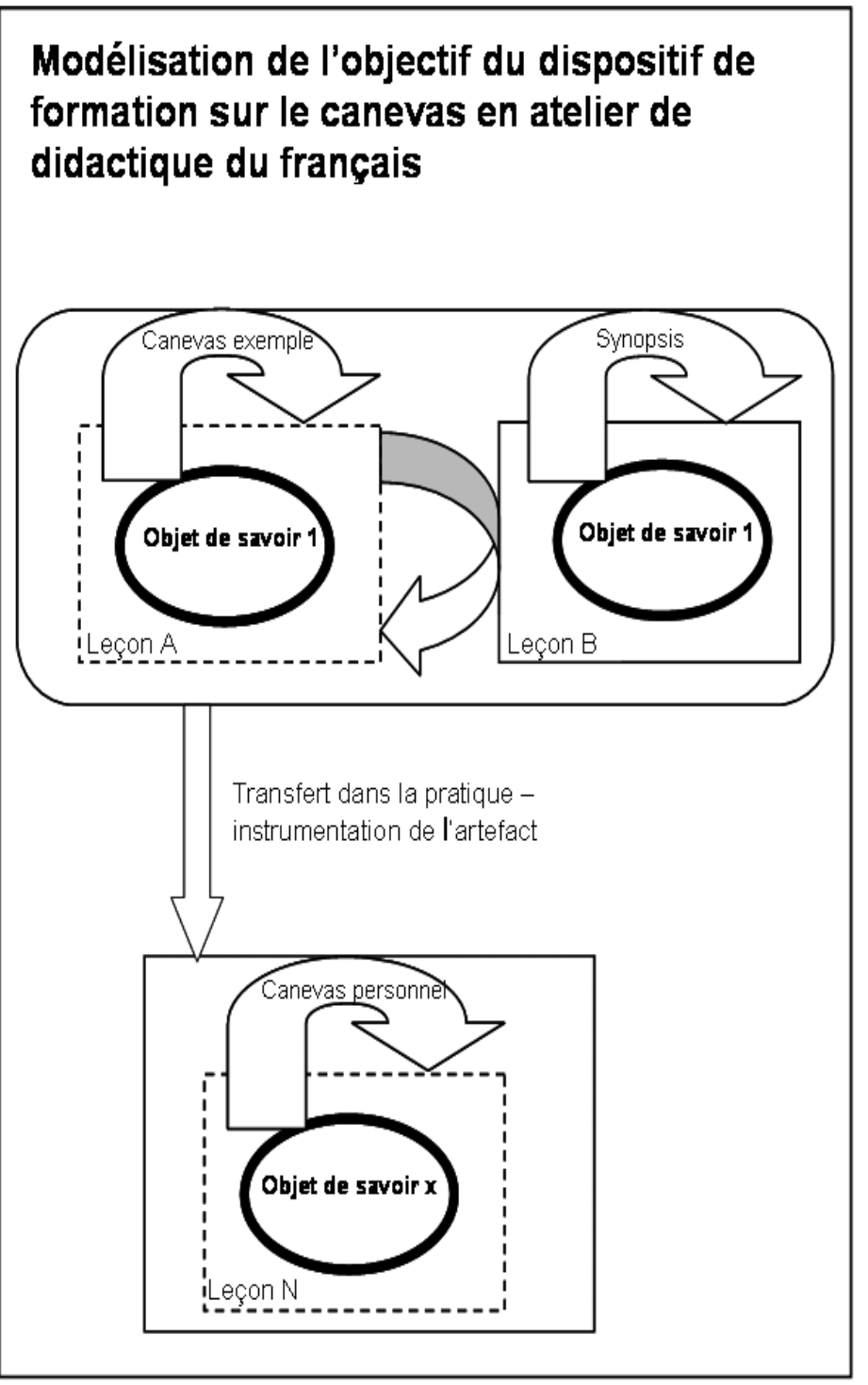

\section{Modélisation de l'objectif du dispositif de formation sur le canevas en atelier de didactique de la physique}

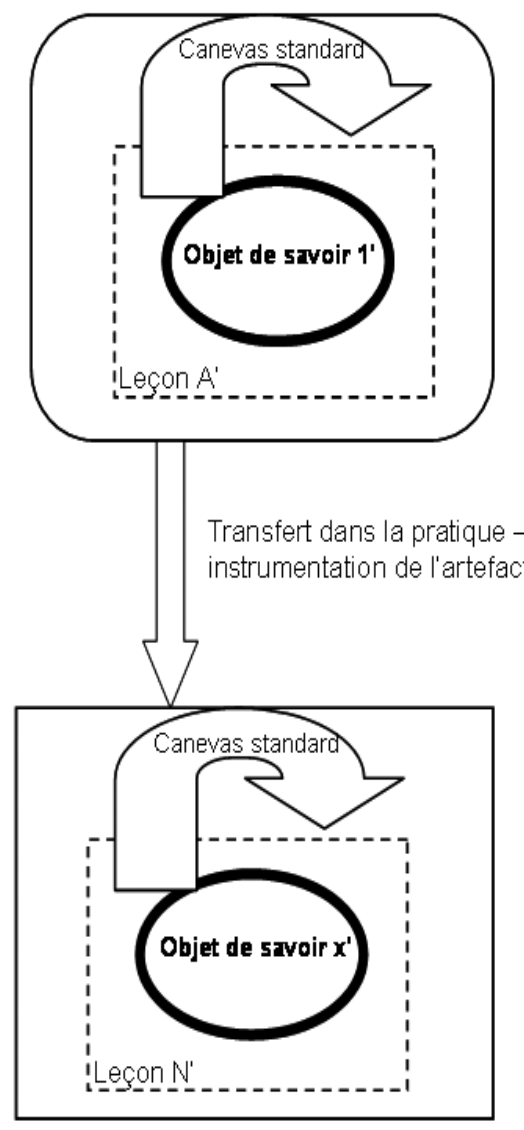

Schéma 1: Double dispositif de formation

Notre dispositif parallèle est donc construit autour d'un travail de préparation d'un enseignement de ces objets de savoir à enseigner - subordonnée relative et masse volumique - à l'aide d'un outil pour enseigner - le canevas de leçon. Cette façon de procéder nous permet de poser l'hypothèse suivante.

\section{Hypothèse}

Cette recherche s'intéresse à la transformation d'un artefact - ici le canevas de leçon - en un instru- ment professionnel, c'est-à-dire à l'actualisation de l'artefact dans un usage finalisé (Rabardel, 1995). À la suite de Riff et Durand (1993), nous partons du postulat selon lequel, dans la mesure où les enseignants novices ne possèdent pas les routines nécessaires pour une activité anticipatrice de planification, il est nécessaire en formation initiale de travailler des outils de planification, non seulement pour le moyen et le long terme, mais également pour le court terme.

À partir de là, nous cherchons à voir, dans la ligne de Tricot et al. (2003), si l'outil canevas de leçon, 
tel qu'il a été abordé, d'une part dans le dispositif avec les enseignants de français, d'autre part dans celui avec les enseignants de physique, est acceptable, utilisable et utile pour les étudiants. Nous posons ensuite l'hypothèse selon laquelle le dispositif de français, articulant le canevas de leçon - qui se construit a priori - au synopsis - outil issu de la recherche en didactique du français qui décrit et analyse la logique d'ensemble de l'action didactique a posteriori - permet mieux aux étudiants d'intégrer les savoirs à enseigner et les savoirs pour enseigner de leur discipline que le dispositif de physique où le canevas est travaillé pour lui-même en lien avec plusieurs concepts de didactique des sciences.

\section{Données et méthodologie}

Pour mettre à l'épreuve cette double hypothèse, nous privilégions une démarche qualitative s'appuyant sur deux types de données permettant d'approcher l'objet selon des angles différents - ce qui constitue d'ailleurs un des points forts de cette recherche: canevas et comptes rendus, établis soit par les étudiants, soit par les formatrices, portant sur des sujets divers en français et en physique. Dans ce contexte, il convient de se référer à Bourdieu (1995) qui rappelle que si le rôle de la science est de se déprendre du local pour aller vers l'universel, l'insertion locale d'une recherche est une condition d'existence et de pertinence. Comment cependant délimiter un champ d'investigation à la fois sur le plan temporel et spatial qui permette ensuite, sans nécessairement aller vers l'universel, de tirer un certain nombre de conclusions dépassant le contexte particulier? Il s'agit pour nous d'analyser, puis de mettre en contraste des données recueillies dans l'atelier de français et sur le terrain auprès de six étudiants avec celles recueillies dans l'atelier de physique et sur le terrain auprès de quatre étudiants. Précisons encore que certaines des données que nous avons récoltées sont "invoquées " (Van der Maren, 1999) - c'est le cas des dix-huit canevas de leçons rédigés par les étudiants de physique et des dix canevas de français, ainsi que des trente comptes rendus d'observation sur le terrain pour le français et des dix-huit comptes rendus pour la physique, et que d'autres sont « suscitées »- treize questionnaires complétés par les étudiants suivis sur le terrain et par quelques autres étudiants des deux ateliers.

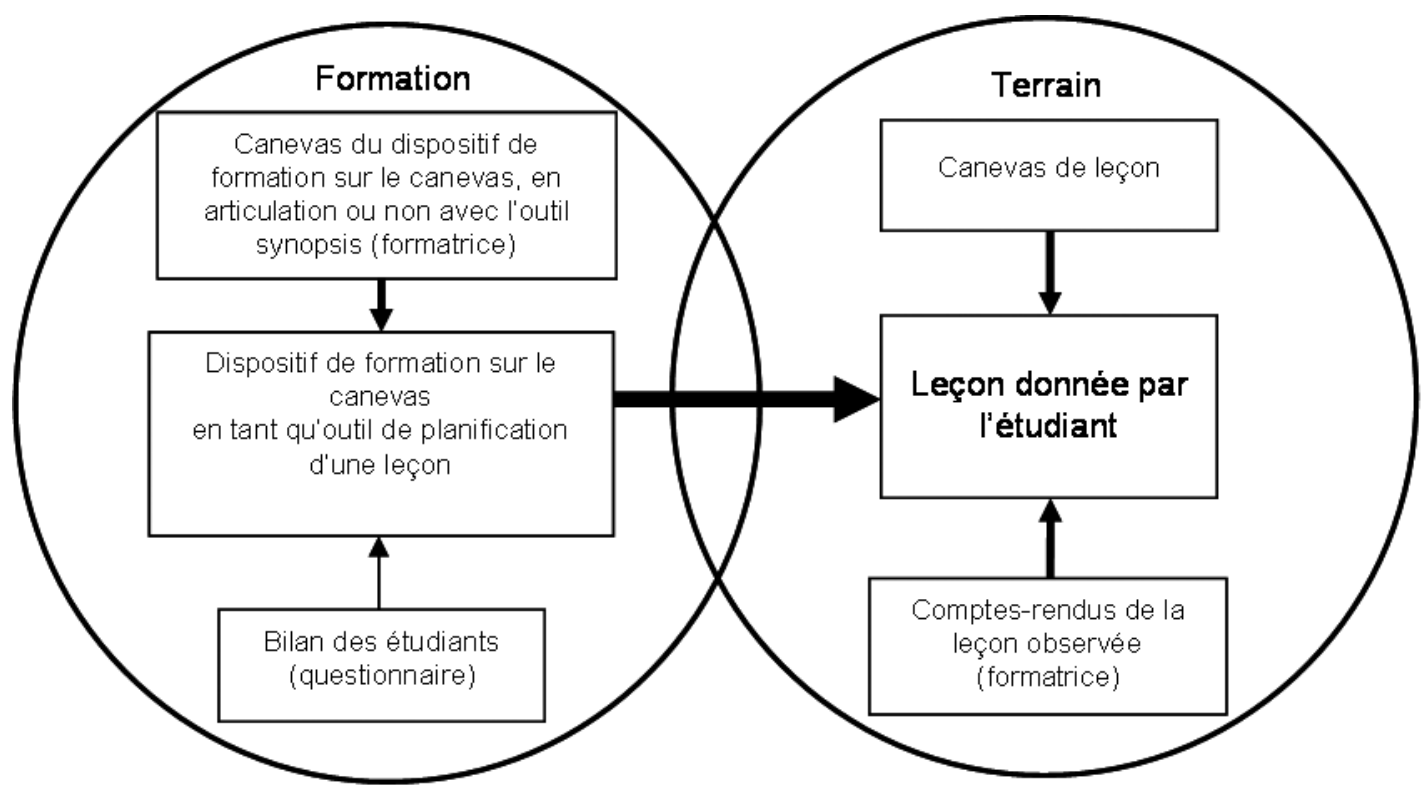

Schéma 2: Données analysés par les chercheuses. 
En nous inspirant des critères de Tricot et al. (2003), nous définissons pour nos données les critères suivants. Pour l'acceptabilité, nous cherchons à voir si l'outil canevas est en adéquation avec les valeurs et la façon d'enseigner des étudiants, à l'aide des réponses données au questionnaire d'évaluation, mais aussi des comptes rendus des leçons observées. Lutilisabilité de l'outil dans chaque dispositif est évaluée à partir de l'analyse des canevas élaborés par les étudiants et des réponses au questionnaire, en fonction d'une part de la compréhension par les étudiants des rubriques de l'artefact et de leur signifiance, d'autre part de la charge de travail. Enfin, l'utilité du canevas est mesurée empiriquement par le biais des comptes rendus des leçons observées à l'aide des critères suivants : comparaison de leçons avec ou sans canevas du point de vue de leur structure, de la précision et de la présentation des objectifs d'enseignement-apprentissage, adéquation de ceux-ci aux contenus et aux activités prévues.

\section{Déroulement des séances sur le canevas en didac- tique de la physique et en didactique du français}

En didactique de la physique, l'atelier sur le canevas s'est déroulé selon des modalités classiques: sur la base d'un travail de préparation individuel ${ }^{3}$, les étudiants ont comparé en groupes de deux ou trois personnes leurs préparations et ont rédigé une version collective qu'ils ont présentée à leurs collègues. La formatrice a alors synthétisé les éléments de leur planification et les a enrichis d'une présentation théorique de différents concepts et outils utiles pour la planification de l'enseignement. Se recentrant sur le canevas de leçon, elle en a évoqué plusieurs formes possibles en interaction avec les étudiants pour mettre en évidence certains avantages et inconvénients. Une forme «standard » a enfin été proposée, illustrée par le canevas d'une leçon sur l'introduction de la notion de masse volumique:

Données contextuelles: école, classe, date, heure, effectif

Thème (notion à introduire ou à consolider)

Prérequis

Objectifs d'apprentissage spécifiques à la leçon (représentation initiale à faire évoluer, obstacle conceptuel à affronter, savoir essentiel, élargissement du champ de signification d'un concept, apprivoisement et stabilisation de connaissances, etc.)

Progression et structure de la leçon

\begin{tabular}{|c|c|c|c|c|c|}
\hline Tâches scolaires $^{4}$ & Temps & $\begin{array}{c}\text { Phases de l'activité } \\
\text { en classe }\end{array}$ & FST & Activités des élèves & $\begin{array}{c}\text { Activités de } \\
\text { l'enseignant }\end{array}$ \\
\hline
\end{tabular}

Tableau 1: Canevas standard proposé aux étudiants de physique

On peut noter dans la parenthèse qui suit les « objectifs d'apprentissage spécifiques » les mentions « représentation initiale à faire évoluer » et " obstacle conceptuel à affronter ». La représentation initiale fait référence aux préconceptions des élèves, qui s'érigent souvent en obstacles à l'apprentissage en sciences. Le concept d'obstacle n'est pas toujours facile à comprendre par les étudiants, car ils le confondent souvent avec de simples difficultés liées à la tâche et aux modalités de travail. Il semble donc important de les rendre attentifs, lors de la planification de la leçon, aux éventuels blocages des élèves face aux obstacles inhérents à la matière étudiée, et de les inviter à prévoir des situations-problèmes pour permettre aux élèves de les affronter ainsi qu'à anticiper d'éventuels étayages pour faciliter leur dépassement. Cette façon de faire permet aussi de définir de façon plus opérationnelle les objectifs spécifiques de la leçon en fonction des obstacles à dépasser. Un autre élément à relever dans ce canevas est le sous-découpage des tâches scolaires en phases, qui oblige les étudiants à penser une structure de la leçon, découpée en moments clairement différenciés avec des rôles bien définis pour les élèves et l'enseignant. Enfin, on constate dans ce canevas des colonnes différentes pour la tâche scolaire (le savoir), les activités des élèves (l'élève), les activités de l'enseignant (le maître) invitant l'utilisateur de cet artefact à distinguer les trois pôles du contrat didactique (Brousseau, 1998). 
Le dispositif élaboré en français est légèrement différent par le fait qu'il articule le canevas au synopsis. Dans un premier temps, par petits groupes, les étudiants ont élaboré un projet de leçon sur l'enseignement de la subordonnée relative. Ils ont ensuite visionné le début d'une séquence dispensée par un enseignant expérimenté sur ce même sujet, destinée à des élèves de 14 ans. Cette séquence, qui travaille la subordonnée relative en lien avec la compréhension et la production de descriptions littéraires et non littéraires, rejoint de ce point de vue les propositions didactiques de Chartrand (2009). À partir de là, les étudiants ont élaboré le synopsis de cette première leçon (voir annexe) sur la base des indications données par la formatrice, en vue d'une confrontation avec leur propre projet de leçon. Dans un troisième temps, l'outil canevas de leçon a été défini, et illustré par la reconstitution, par la formatrice, du canevasprojet de l'enseignant filmé à partir des informations recueillies lors de l'entretien avec ce dernier.

Notons que le canevas proposé est quelque peu différent de celui proposé en didactique de la physique. Conçu comme l'envers du synopsis, il en reprend les rubriques principales, qui placent au centre le déploiement du savoir, ne laissant qu'une seule colonne pour les deux autres acteurs, les élèves et l'enseignant (Dolz, Schneuwly, \& Ronveaux, 2006).

Données contextuelles: école, classe, date, heure, effectif:

Thème (notions et/ou démarches à introduire ou à consolider):

Objectifs d'apprentissage spécifiques:

Institutionnalisation prévue:

Inscription de la leçon dans le programme:

Déploiement de l'objet de savoir:

\begin{tabular}{|c|c|c|c|c|}
\hline $\begin{array}{c}\text { Niveau (de } \\
\text { déploiement du } \\
\text { savoir) }\end{array}$ & Minutage & FST & Matériel & $\begin{array}{c}\text { Activités des élèves/Modalités } \\
\text { d'intervention de l'enseignant }\end{array}$ \\
\hline
\end{tabular}

Tableau 2: Canevas proposé aux étudiants de français

Ainsi, par opposition au canevas de physique, il n'y a pas la rubrique « prérequis », ni d'indications concernant les obstacles à identifier, puisque ces concepts ne sont pas développés dans le synopsis. De même, on ne trouve pas la référence aux phases de la tâche. Seule a été retenue, en lien avec les « objectifs d'apprentissage spécifiques », la phase d'institutionnalisation qui, bien que provenant de la didactique des mathématiques (Chevallard, 1985), peut être transposée en didactique du français (Forget, 2008). Enfin, la mention de l'inscription de la leçon dans le programme incite les étudiants à concevoir une leçon comme une partie d'un tout plus vaste, la séquence d'enseignement.

À partir de là, comment, en fonction des deux dispositifs, l'outil canevas a-t-il été accepté, manié et utilisé par les étudiants de français et de physique?

\section{Présentation des résultats}

\section{De l'acceptabilité du canevas}

Pour évaluer l'acceptabilité de l'outil, nous cherchons à voir si le canevas ne s'oppose pas aux valeurs des étudiants et à leur manière d'enseigner. Pour ce faire, nous nous appuyons pour le premier point sur les réponses données au questionnaire d'évaluation, pour le second sur les comptes rendus des leçons observées avant le dispositif.

Du point de vue des valeurs, il semble que l'outil soit tout à fait acceptable. En effet, aucun étudiant, qu'il soit de français ou de physique, n'évoque dans les réponses au questionnaire l'idée que le canevas puisse être aliénant ou qu'il l'ait 
contraint à une démarche en opposition avec ses valeurs. En revanche, à propos de la façon d'enseigner des enseignants en début de formation, certains indices nous laissent penser que le canevas peut correspondre ou au contraire s'opposer à la manière d'enseigner de ces derniers. Lanalyse des comptes rendus de leçons de français comme ceux de physique met en effet en évidence le fait que certains enseignants, avant même d'avoir suivi le dispositif sur le canevas, ont visiblement une structure précise de la leçon en tête et suivent un timing: ils regardent leur montre, arrêtent une activité après un certain temps, et sont très soucieux d'impulser un rythme à la leçon. Une étudiante de français fait même un canevas personnel dès le début de l'année. D'autres, au contraire, laissent spontanément plus de place aux interventions des élèves, et vont jusqu'à leur conférer la responsabilité d'insuffler le rythme de la leçon.

Autrement dit, il semble bien que l'artefact proposé soit plus difficilement acceptable pour certains que pour d'autres. Dans leurs réponses au questionnaire, certains avouent en effet que, pour eux, préparer un cours à l'aide d'un canevas est « difficile, car il faut anticiper le déroulement chronologique de la leçon ». Pour d'autres, c'est plutôt l'utilisation du canevas au moment du cours qui leur pose problème, dans la mesure où il les empêche de prendre en compte la dynamique de classe: «Il ne doit pas non plus être un frein à une gestion dynamique et intelligente de chaque cours, avec les innombrables spécificités propres à chaque classe ou groupe d'élèves ». Ces phrases peuvent être interprétées comme la découverte en acte de l'importance de la chronogenèse, c'est-à-dire du fait que l'avancement du temps d'apprentissage n'est pas aussi linéaire que celui du temps didactique.

\section{De l'utilisabilité du canevas}

Pour évaluer l'utilisabilité de l'outil, nous nous intéressons à la compréhension par les étudiants des différentes rubriques de l'artefact et à leur signifiance, ainsi qu'à la charge de travail qu'il leur occasionne. Pour le premier point, nous nous appuyons sur les canevas élaborés par les étudiants, pour le second, sur les réponses données au questionnaire d'évaluation.
En physique, une observation de leçon a eu lieu pour chaque étudiant avant l'atelier, pour laquelle il leur a été demandé d'établir un canevas à leur idée. Or, l'analyse de ces documents montre que les étudiants ont déjà une représentation assez proche du modèle qui leur sera proposé ultérieurement. Ainsi, le canevas de l'un d'entre eux contient, après les informations de type contextuel, sous le titre " description de la séquence ", l'indication de l'objectif d'enseignement et des objectifs d'apprentissage pour les élèves, rédigés sous la forme "les élèves devraient acquérir...» suivis par la liste des prérequis et du matériel. Sous le titre "déroulement de la séquence ", ce canevas renvoie au cours rédigé par l'enseignant et en propose un découpage entre les quatre périodes de 45 minutes prévues pour ce sujet. Les autres canevas libres sont un peu différents, mais tous comportent les objectifs d'apprentissage pour les élèves. Deux canevas présentent un tableau à double entrée séparant les différentes étapes de la leçon, préfiguration des phases, et deux proposent un découpage temporel relativement précis. Notons aussi que deux étudiants prévoient de leur propre chef des indications séparées pour les activités du maître et des élèves. Enfin, un étudiant identifie des obstacles à l'apprentissage des élèves dans l'objet de savoir de sa leçon.

Lanalyse des treize canevas de physique élaborés suite à l'atelier fait état d'une prise en main progressive des différentes rubriques. En particulier, les objectifs sont non seulement formulés plus clairement, sous forme de connaissances et de compétences à atteindre par les élèves, mais sont surtout plus en cohérence avec les activités proposées à ces derniers. De même, la colonne timing est remplie par tous à la minute près, ce qui n'empêche pas l'étudiant $\mathrm{D}$ de s'en détacher librement pendant la leçon, en fonction des interactions avec les élèves. Quant aux colonnes « activité de l'enseignant » et « activités des élèves », elles ne posent pas de problème de compréhension, même si elles n'incitent pas forcément les étudiants à dévoluer davantage certaines tâches aux élèves: on peut lire par exemple dans cette dernière colonne une suite de "se taisent", " écoutent", « copient au tableau ». Pour l'auteur de ce canevas, le rôle des élèves est avant tout celui d'un public qui assiste à sa leçon, montrant une vision topogenétique initialement unimodale, qui évolue dans les canevas successifs. De même, la colonne « activité de l'enseignant » est surtout utilisée pour des indications 
de gestion de la classe, comme « s'assurer que les élèves travaillent bien » et pour préciser les consignes " poser la situation-problème: comment savoir si deux échantillons sont de la même substance? ", ou encore pour décrire le savoir visé à la fin des activités, ce qui correspond à des éléments d'institutionnalisation. D'autres étudiants indiquent plutôt le texte du savoir dans les deux premières colonnes concernant les tâches scolaires et les phases. À ce stade, l'utilisation différente de ces colonnes, loin d'être le signe d'une utilisation inadéquate de l'outil, met en évidence un début d'instrumentation de ce dernier selon la définition de Rabardel (1995).

Deux rubriques posent néanmoins problème. Un certain flou persiste dans le concept de tâches scolaires et phases de ces tâches. Ainsi cette colonne contient souvent des informations plutôt de l'ordre de la gestion de classe, alors qu'on attendait la définition de tâches visant l'acquisition d'un savoir. C'est le cas également des obstacles, où on trouve plutôt des listes de difficultés des élèves. Comment interpréter ces confusions, alors que la représentation initiale du canevas proche de l'outil proposé aurait laissé penser que son utilisabilité serait relativement aisée? Ne serait-ce pas dû d'une part à l'abstraction de ces concepts didactiques et d'autre part à l'important bagage scientifique des étudiants qui rend plus difficile le repérage des obstacles du savoir?

Qu'en est-il maintenant des canevas des enseignants de français pour lesquels l'introduction de l'outil a été articulée à l'outil de recherche synopsis? Comme il n'a été demandé aux étudiants de français qu'un seul canevas, nous n'avons à disposition que dix documents, un produit par chacun des six étudiants suite au dispositif, plus quatre autres qu'une étudiante a pris l'initiative de rédiger pour chaque leçon observée. D'abord, alors qu'en français aucun modèle n'a été imposé, les étudiants reprennent tous la forme du modèle proposé en exemple en atelier, à l'exception de cette étudiante qui maintient sa manière personnelle de fonctionner et remplace le tableau à double entrée par un texte rédigé. Les titres donnés au document divergent cependant d'un étudiant à l'autre: si trois étudiants parlent de "fiche de préparation ", montrant par là même qu'ils confèrent une finalité précise au canevas et l'instrumentalisent pour reprendre les termes de Rabardel (1995), deux étudiants reprennent le terme de « canevas », et un étudiant utilise de façon erronée le terme de « synopsis», laissant penser qu'il confond les deux outils.

À partir de là, on peut faire un certain nombre de constats concernant le contenu. D'abord, les étudiants remplissent toutes les cases proposées, et plusieurs rubriques sont parfaitement maîtrisées. C'est le cas du thème, de l'inscription dans le programme, ainsi que des colonnes concernant le timing, le matériel, et les formes sociales de travail. D'autres rubriques semblent cependant poser problème. La première est celle des « objectifs d'apprentissage spécifiques » que certains ont de la peine à formuler. Ainsi, un étudiant place sous cette rubrique la liste suivante: « approche de la poésie et de ses spécificités; compréhension et analyse de texte; savoir repérer une comparaison et son utilisation; savoir repérer les pronoms personnels et démonstratifs et leurs utilités; approche de la notion de lyrisme ». Or, mis à part le fait que cette liste s'apparente à un embryon de programme qu'il est impossible de couvrir sur 90 minutes, il convient de relever que les cinq « objectifs » de cette liste ne sont pas sur le même plan: les troisième, quatrième et cinquième objectifs pourraient notamment être vus comme des "sous-objectifs» du deuxième " objectif », lui-même vu comme un moyen pour aborder le premier « objectif ». La deuxième rubrique qui fait problème est « l'institutionnalisation ». Au lieu d'essayer d'anticiper ce qui devra être validé par l'enseignant en fin de leçon, les étudiants se limitent à réécrire les objectifs d'enseignement de la leçon, ou alors remplacent l'institutionnalisation par une citation d'un manuel du maître. La troisième rubrique qui met les étudiants en difficulté est celle des « niveaux ». Dans le synopsis détourné (Monnier \& Weiss, 2009), le niveau 0 indique soit une parenthèse, soit une incise par rapport au déploiement du savoir, le niveau - $\mathrm{n}$ désigne un objet de savoir de la leçon, le niveau $-\mathrm{n}-\mathrm{n}$, l'activité de l'enseignant en lien avec cet objet savoir, le niveau $-\mathrm{n}-\mathrm{n}-\mathrm{n}$, les activités des élèves en lien avec cet objet (voir annexe). Or, si certains étudiants ont compris le principe et le réinterprètent à leur manière - c'est le cas notamment de l'étudiante $\mathrm{P}$ qui, ayant deux objets de savoir distincts, l'un de contenu, l'autre méthodologique, simplifie le principe en se limitant à lister les activités de l'enseignante et celles des élèves par rapport à ces deux savoirs, d'autres proposent un découpage arbitraire et qui ne semble obéir à aucune logique. Enfin, la colonne de droite qui, articulée à la colonne des 
niveaux, comprend, soit les activités des élèves, soit les interventions de l'enseignant sème la confusion auprès des étudiants. N'ayant pas compris que ces deux colonnes, niveau et description, sont interdépendantes, ils remplissent cette dernière à partir de leur conception de l'enseignement avant tout transmissive, ce qui a pour conséquence une centration importante sur les interventions de l'enseignant au détriment des activités élèves pour lesquelles on trouve essentiellement "écouter », "s'exercer » et « copier».

Comment expliquer ces difficultés? Plusieurs raisons peuvent être avancées. D'abord, les étudiants sont au début de leur formation et n'ont pas encore eu le temps d'intégrer tous les concepts didactiques sousjacents au canevas, en particulier ceux d' ' objectif d'apprentissage » et d' « institutionnalisation », d'autant que la transposition de ce concept en didactique $\mathrm{du}$ français pose certains problèmes du point de vue du rapport à la vérité, comme le souligne Forget: « Le rapport à la vérité est central en mathématiques. [...] C'est par le processus de preuve (permettant de valider une proposition) que l'élève peut accéder à la vérité mathématique. [...] En ce qui concerne la discipline du français, nonobstant son caractère éminemment social, l'enjeu est d'un autre ordre: c'est par l'argumentation et/ou par la convocation de normes culturellement instituées que l'on cherche à approcher la vérité » (Forget, 2008, p. 81). Ensuite, contrairement aux étudiants en physique qui ont eu l'occasion de travailler le canevas à plusieurs reprises sur des objets de savoir différents, les étudiants de français se sont limités à un premier essai.

Enfin, en ce qui concerne la charge de travail en lien avec l'utilisabilité de l'outil, les commentaires des étudiants, qu'ils soient de français ou de physique, sont de deux types: certains avouent y renoncer quand il n'y a pas la visite de la formatrice par manque de temps: « jamais, car je n'en aurais pas le temps », d'autres au contraire disent l'utiliser, mais de façon simplifiée, ce qui constitue pour nous un signe d'instrumentalisation de l'artefact: « toujours, mais sans indiquer de manière très détaillée la durée de chaque tâche».

Ainsi, bien que les étudiants - en particulier ceux de français - aient certaines difficultés à comprendre les différentes rubriques du canevas, et que plusieurs admettent ne pas l'utiliser dans leurs préparations quotidiennes par manque de temps, plusieurs laissent entendre que non seulement ils considèrent l'outil utilisable, mais encore qu'ils l'ont bien transformé en un instrument efficient leur permettant de structurer leur cours de façon efficace.

\section{De l'utilité du canevas}

Enfin, nous tentons de déterminer l'utilité du canevas au sens de Tricot et al. (2003) du point de vue de l'efficacité pour l'enseignement en comparant les comptes rendus des leçons observées, selon que les étudiants ont élaboré ou non un canevas, et en regardant ce qu'ils en disent dans leurs réponses au questionnaire. C'est principalement dans le corpus des leçons de français que nous pouvons observer des écarts entre les leçons préparées à l'aide d'un canevas et celles pour lesquelles l'enseignant n'en avait pas fait, les leçons de physique étant toujours accompagnées de canevas. Pour rappel, les comptes rendus d'observation en français concernent trente leçons dont cinq ont été données avant l'atelier sur le canevas. Pour l'analyse, deux paramètres sont à prendre en compte. D'abord, le moment de la visite dans l'année, puisqu'il s'agit d'enseignants débutants qui gagnent en expérience au cours du temps. Ensuite, le fait que certaines leçons observées ont été précédées par l'élaboration d'un canevas, et c'est la variable qui nous intéresse ici.

Si on relit les comptes rendus des leçons observées avant le dispositif de formation, on remarque que ces leçons ne sont pas organisées autour d'objectifs d'enseignement-apprentissage précis. En effet, chez ces six enseignants en formation initiale, les leçons touchent souvent plusieurs domaines distincts, abordés successivement, sans que des liens soient explicités. Ainsi, un enseignant choisit d'aborder en première heure la lettre commerciale et en deuxième heure la notion de personnage; un autre propose pour les deux heures une batterie d'exercices portant sur des savoirs différents, le vocabulaire, l'argumentation, l'orthographe, et qui ne portent pas sur le même support. Tout se passe en fait comme s'il fallait pouvoir cocher les différentes notions prescrites par le plan d'études, sans préoccupation d'inscrire les activités proposées dans une logique qui puisse faire sens pour les élèves, comme cela est pourtant proposé 
par ces mêmes plans d'études. Corollairement, aucun des enseignants observés ne présente d'objectif d'apprentissage aux élèves, très peu proposent des activités-élèves et celles-ci sont rarement délimitées dans le temps, enfin pratiquement aucun support n'est utilisé, que ce soit des questionnaires-guide, des consignes écrites, des documents à compléter, ni même des moyens visuels aussi simples que le tableau noir ou le rétroprojecteur.

Lanalyse des comptes rendus des leçons observées après le dispositif de formation met en évidence plusieurs changements. Si tous les étudiants ne font pas systématiquement un canevas, ils prennent visiblement davantage en compte ses différentes rubriques, notamment celles du timing, du matériel, et des activités des élèves. Autrement dit, la réflexion sur le canevas semble aider les étudiants à penser la leçon en termes de tâches pour les élèves et non plus exclusivement en termes de contenus à maîtriser. Néanmoins, la définition d'objectifs d'apprentissage précis et l'articulation de ceux-ci aux tâches proposées reste une difficulté importante. Ainsi, alors qu'elle a annoncé en début d'heure aux élèves que la leçon porterait sur la rédaction d'un commentaire composé sur un poème précis, une étudiante propose aux élèves de se mettre par groupes de quatre et de faire une analyse comparative de trois poèmes. Cette activité, tout en étant évidemment en lien avec l'objectif annoncé - l'un des trois étant celui qui fera l'objet d'un commentaire composé dans un second temps, est déstabilisante pour les élèves qui n'en voient pas le sens.

En comparant maintenant, parmi le corpus de leçons données par chaque étudiant après l'atelier, celles accompagnées de canevas avec celles sans canevas, on constate que cet outil constitue une aide pour la construction de leçons plus structurées avec un fil conducteur plus clair, laissant moins la porte ouverte à des difficultés de gestion de classe. Le cas d'une étudiante est d'ailleurs emblématique à ce propos: alors qu'elle était parvenue, lors de la deuxième leçon observée pour laquelle elle avait fait un canevas, à se détacher du cours ex cathedra et à prévoir des tâches pour les élèves avec un timing précis, elle laisse ultérieurement tomber le canevas. La troisième leçon observée est parasitée cependant par un certain nombre de problèmes de gestion de classe, et pour la quatrième leçon observée, elle revient d'elle-même au canevas.
Ainsi, l'utilité du canevas pour des enseignants débutants réside en priorité dans l'incitation à une anticipation au niveau du triplet mésogenèse, topogenèse et chronogenèse de la leçon, c'est-à-dire non seulement du point de vue des contenus à enseigner, mais aussi du lien entre les objectifs d'apprentissage et un scénario comportant des activités, un timing et des modalités de travail. Pour certains, le canevas permet d'anticiper les problèmes: « ça m'a forcé à tout anticiper, à ne pas laisser de flou »; pour d'autres, l'intérêt de l'outil réside avant tout dans la réflexion qu'il incite à faire sur les avantages et les inconvénients de tel ou tel choix didactique: « il m'a permis de mieux préparer mes leçons, d'être conscient de la nécessité de justifier les différents choix (didactiques, méthodologiques,...) que je fais »; d'autres encore mettent en évidence la possibilité, grâce à cet outil, de garder le fil conducteur au moment du cours: « il permet de garder en tête l'objectif, malgré les chemins sinueux où parfois peuvent nous mener les questions des élèves », et, après le cours, d'avoir un regard réflexif sur sa propre pratique: « un canevas bien fait permet de comparer précisément notre préparation de la leçon et son déroulement effectif, et cela est d'autant plus important pour un nouvel enseignant. Il nous permet de (et oblige à) réfléchir sur notre pratique, en mettant par écrit nos attentes et nos analyses, pour les comparer par la suite à ce que le cours a effectivement donné ». Autrement dit, la plupart des étudiants considèrent cet outil comme essentiel pour un enseignant novice: " au moment de la leçon, le canevas est d'une grande aide. Il me permet de mener une leçon parfaitement structurée. Il faudrait que le canevas devienne l'un des outils indispensables de l'enseignant », tout en soulignant la nécessité de s'en détacher au moment de la leçon: " comme le nom le dit (« canevas»), il existe pour s'en départir », ce qui constitue pour nous un nouvel indice de l'appropriation par les étudiants de l'outil qu'ils transforment en un instrument opérationnel pour leur enseignement en classe.

\section{De la pertinence du synopsis en formation}

À partir de là, il convient d'éprouver le dispositif de formation de français en se posant les questions suivantes: le détour par le synopsis, dont la stucture est relativement proche de celle du canevas, contribue-t-il à ce que les étudiants comprennent et utilisent mieux les différentes rubriques du canevas? 
Le travail prospectif de préparation d'une leçon sur la subordonnée relative articulé à un travail rétrospectif de description et d'analyse d'un extrait d'une séquence sur ce sujet par un enseignant expert encourage-t-il les étudiants à aborder ce sujet avec les élèves, en particulier lorsqu'ils sont observés sur le terrain par leur formateur? Enfin, l'analyse, en atelier, des écarts entre plusieurs possibles - les canevas des étudiants - et l'actualisation d'un possible - la leçon filmée de l'enseignant expert décrite et analysée par les étudiants par le biais de l'élaboration du synopsis - aide-t-elle les étudiants à adopter une démarche réflexive vis-à-vis de leur propre pratique?

En reprenant nos analyses des dix canevas des leçons de français et des dix-huit canevas de physique par rapport à l'utilisabilité de l'outil et en comparant les deux groupes de canevas par discipline, force nous est de reconnaître que, loin de constituer une aide pour la compréhension du canevas, le détour par le synopsis a été plutôt perturbant pour les étudiants de français. En effet, même si l'obligation de faire un canevas pour chaque leçon observée a donné l'occasion aux étudiants de physique de se pencher à plusieurs reprises sur cet outil, leur permettant sans doute de dépasser certaines difficultés, ces étudiants semblent avoir eu globalement moins de problèmes à comprendre les différentes rubriques de l'outil qui leur a été imposé. Au contraire, en français, les deux rubriques empruntées directement au synopsis ont posé de sérieux problèmes aux étudiants qui n'ont pas réussi à découper le savoir par niveaux en articulation avec les activités prévues des élèves et les interventions anticipées de l'enseignant. De plus, il semble qu'ils n'aient pas tous saisi que le synopsis est toujours construit a posteriori par opposition au canevas qui, lui, l'est forcément a priori; c'est notamment le cas de l'étudiant qui a utilisé le titre « synopsis» au lieu de celui de « canevas ».

Si le dispositif de français n'a pas eu de répercussions positives au niveau de l'utilisabilité de l'outil, on pourrait penser que la réflexion autour de l'enseignement de la subordonnée relative, renforcée par l'observation d'un enseignant expert, aurait incité les étudiants à expérimenter avec leurs élèves le sujet traité en atelier. Pourtant, tel n'est pas le cas. Alors que le dispositif portait sur la grammaire de phrase, aucune des trente leçons observées ne porte sur cet objet de savoir. Comment comprendre cela?
Notre explication s'appuie sur plusieurs entretiens avec les enseignants en formation de français. Lors d'une visite du formateur en classe, les enseignants en formation pensent qu'il est préférable de choisir un sujet plus « élevé » et plus "intéressant » qu'un objet grammatical, et cela pour deux raisons; il s'agit pour eux d'abord de préparer un cours qui « séduira » le formateur - d'où une proportion importante de cours portant sur la littérature, et non sur la grammaire connotée comme plus utilitaire; il s'agit ensuite d'adopter un sujet qu'ils maîtrisent particulièrement bien, ce qui n'est pas toujours le cas pour la grammaire. Plusieurs d'entre eux nous ont en effet avoué ne pas être à l'aise avec l'enseignement de la grammaire rénovée. Comme nous l'avons déjà souligné - et cela doit interroger la formation - les enseignants du secondaire n'ont la plupart du temps que des souvenirs de grammaire qui remontent à leur scolarité primaire ou secondaire obligatoire, et certains n'ont jamais été initiés à la grammaire rénovée. Ensuite, le fait que les étudiants soient encouragés en formation à travailler sous forme de séquences les incite peutêtre à mettre entre parenthèses - surtout au secondaire post-obligatoire - les notions et compétences grammaticales, ou à travailler ces points de façon décloisonnée à l'aide d'exercices trouvés dans différents manuels. Ces constats rejoignent donc ceux d'Elalouf et Péret pour la France (2009): sur 700 inspections faites entre 2002 et 2007, seules seize séances d'étude de la langue ont été observées, soit un pourcentage de 2,3\%. Et ces chercheurs d'avancer l'explication suivante: «le passage d'un enseignement cloisonné en différentes sous-disciplines à un enseignement par séquence s'est fait au collège au dépens de l'étude de la langue, d'autant que les programmes reléguaient celle-ci au rang d'outil » (Elalouf \& Péret, 2009, p. 51).

Est-ce à dire alors que le détour par le synopsis, au lieu d'être un facilitateur, a constitué une entrave à l'acquisition par les étudiants des savoirs à et pour enseigner visés par le dispositif? Pas tout à fait. Il nous semble que si l'outil « canevas » a été plus difficile à comprendre et à utiliser pour les étudiants de français que pour ceux de physique, c'est parce que, articulé au synopsis, le canevas se voit conférer une facette conceptuelle plus importante. En particulier, en mettant au second plan le déroulement chronologique, le synopsis apporterait au canevas le côté " narration » en épisodes et sous-épisodes (les niveaux) centrés avant tout sur le déploiement 
du savoir. Mais surtout, sur un autre plan, on peut supposer que le travail d'analyse en petits groupes d'un extrait d'une séquence didactique à l'aide du synopsis favorise chez les étudiants une attitude plus réflexive. En analysant en atelier une leçon d'un enseignant expert, les enseignants en formation vont aiguiser leur regard sur la logique d'ensemble de l'action didactique au niveau d'un extrait d'une séquence d'enseignement filmée, sur des moments et des lieux où se réalisent les gestes professionnels de l'enseignant. Ainsi, loin de constituer un détour qu'on pourrait qualifier d'inutile, l'initiation à l'utilisation d'un outil de recherche issu d'une didactique disciplinaire nous semble très prometteuse pour permettre aux étudiants de développer des compétences d'analyse qu'ils seront susceptibles de transposer dans un troisième temps pour l'analyse de leur propre pratique.

\section{Conclusion}

Nous avons d'abord cherché à voir si l'outil canevas était acceptable, utilisable et utile pour les enseignants en formation dans leur propre pratique. Nous nous sommes rendu compte que si l'acceptabilité du canevas dépend moins du dispositif proposé que des manières d'enseigner des enseignants novices, en tout cas en début de formation, l'utilisabilité de l'outil peut être facilitée par un dispositif de formation qui se limite à associer l'outil professionnel à un certain nombre de concepts didactiques directement utiles pour l'anticipation de la leçon, à l'instar des objectifs, des prérequis, des obstacles et des phases didactiques. Quant à la dimension utilité, elle n'intervient qu'à partir du moment où l'utilisateur parvient à instrumenter l'artefact en fonction de ses besoins. Nous avons ensuite tenté de voir si un dispositif qui croise un travail de planification sur l'enseignement d'un objet précis - l'élaboration d'un canevas - avec un travail de description et d'analyse a posteriori d'une actualisation possible sur le même objet - la construction d'un synopsis - permettrait aux étudiants de mieux s'approprier les savoirs à et pour enseigner. Suite à l'analyse du dispositif de français, force nous est de reconnaître que la construction de ponts entre outils proposés en formation et outils issus de la recherche n'est pas chose aisée. Pourtant, elle constitue selon nous un atout à exploiter à moyen et long terme aussi bien pour les formateurs que pour les chercheurs, parce qu'elle ouvre un dialogue entre deux logiques différentes, mais interdépendantes, comme le relèvent Criblez et Hofstetter (2002). Il reste maintenant à penser et à mettre en action ce transfert.

Revenant ensuite aux préoccupations de la didactique comparée, il conviendrait de s'interroger sur la possibilité et la pertinence de transposer les concepts et outils étudiés dans chacun des ateliers à d'autres didactiques disciplinaires dans le cadre de la formation des enseignants. Pour développer ce champ, nous entrevoyons en tout cas deux pistes possibles. Dans une recherche précédente (Monnier \& Weiss, 2009), nous avons montré qu'il était possible d'utiliser le synopsis pour décrire et analyser une leçon de mathématiques et, dans un travail en cours, nous éprouvons également avec succès cet outil pour une leçon de physique. Poursuivant la logique de mettre des outils de la recherche au service de la formation, nous envisageons d'expérimenter le synopsis dans des ateliers de didactique de la physique, en veillant à éviter les difficultés constatées avec les étudiants de français. Il nous semble en effet que l'intérêt de cet outil est double: d'une part le montrer comme le pendant a posteriori du canevas qui, lui, se construit a priori, d'autre part observer et analyser des leçons filmées d'enseignants experts à l'aide d'un outil d'analyse qui évite les jugements hâtifs, travers qui apparaît souvent dans ce type d'exercice (Monnier et al., 2008) et qui permet de focaliser le regard sur le déploiement du savoir. Une seconde piste nous semble s'ouvrir à partir du concept de phases déjà adapté à la physique par Robardet et Guillaud (1997). Dans quelle mesure et à quelles conditions ces phases pourraient être définies en didactique du français à propos de leçons, en poursuivant la réflexion menée par le GRAFE (Schneuwly et al., 2009) pour l'analyse de séquences d'enseignement? Dans les canevas de physique, les phases n'étaient pas clairement identifiées par les étudiants, et un travail de définition et de clarification de celles-ci semble nécessaire. Pourquoi ne pas proposer aussi ce concept dans le cadre de la formation initiale des enseignants de français, pour leur donner des moyens de mieux structurer leurs leçons au moment de la planification? Le nouvel institut universitaire de formation des enseignants actuellement en place à Genève, conçu sur la base d'une structure interfacultaire, nous semble être un espace de formation susceptible de s'enrichir des apports de la didactique comparée, ouvrant ainsi des perspectives prometteuses pour faire vivre et migrer des concepts et outils d'une didactique à l'autre. 


\section{NOTES}

1. Conférence suisse des directeurs cantonaux de l'instruction publique, organe intercantonal regroupant les autorités des différents cantons suisses en matière d'instruction publique.

2. Loi sur l'instruction publique (LIP) de la République et canton de Genève C 1 10, http://www.ge.ch/legislation/rsg/f/s/rsg_Cl_10.html

3. «Rédiger sur une feuille le déroulement chronologique de l'enseignement de l'introduction de la notion de masse volumique. Longueur: 1 page. Temps de travail pour réaliser la tâche: maximum 1 heure ».

4. Ce terme polysémique a été choisi pour que les étudiants focalisent leur regard sur le travail à faire faire aux élèves. Avec du recul, l'expression « dispositif didactique » proposé par Ligozat (2008) serait peut-être plus approprié.

\section{RÉFÉRENCES}

Astolfi, J.-P. (1997). L'erreur un outil pour enseigner. Paris: ESF.

Astolfi, J.-P. \& Develay, M. (2002). La didactique des sciences. Que sais-je? Paris: PUF.

Astolfi J.-P. \& Peterfalvi, B. (1993). Obstacles et construction de situations didactiques en sciences expérimentales. Aster 16: Modèles pédagogiques 1. 103-141.

Besson, M.-J., Genoud, M.-R., Lipp, B., \& Nussbaum, R. (1979). Maîtrise du français. Méthodologie pour l'enseignement primaire. Neuchâtel: IRDP.

Bourdieu, P. (1995). La cause de la science. Actes de la recherche en Sciences sociales, 106-107, 3-11.

Briand, J. \& Chevalier, M.-C. (1995). Les enjeux didactiques dans l'enseignement des mathématiques. Paris: Hatier.

Brousseau, G. (1998). Théorie des situations didactiques. Grenoble: La pensée sauvage.

Chartrand, S.-G. (2009). Proposition didactique d'une progression des objets à enseigner en français langue première au secondaire québécois. In J. Dolz \& C. Simard. Pratiques d'enseignement grammatical (p. 257-289). Québec: PUL.

Chevallard, Y. (1985). La transposition didactique. Grenoble: La pensée sauvage.

Clerc, F. (1998). Débuter dans l'enseignement. Paris: Hachette.

Criblez, L. \& Hofstetter, R. (2002). La professionnalisation des métiers de l'éducation à travers la tertiarisation de la formation. Revue suisse des sciences de l'éducation, 1, 5-13.

De Pietro, J.-F. (2009). Pratiques métalangagières et émergence d'une posture " grammaticale ». In J. Dolz \& C. Simard. Pratiques d'enseignement grammatical (p. 155-179). Québec: PUL. 
Dolz, J., Schneuwly, B., \& Ronveaux, C. (2006). Le synopsis - un outil pour analyser les objets enseignés. In M.-J. Perrin-Glorian \& Y. Reuter (Ed.). Les méthodes de recherche en didactiques (p. 175-190). Villeneuve d'Ascq: Presses universitaires du Septentrion.

Elalouf, M.-L. \& Péret, C. (2009). Pratiques d'observation de la langue en France: quelles évolutions? quels obstacles? In J. Dolz \& C. Simard. Pratiques d'enseignement grammatical (p. 49-75). Québec: PUL.

Forget, A. (2008). Importer le concept d'institutionnalisation en classe de français: peut-on créer les conditions d'une migration heureuse? Analyse des situations didactiques: perspectives comparatistes, 20, 75-88.

GRAFE (version provisoire du 31.7.05). Rédiger le synopsis d'une séquence d'enseignement. Guide méthodologique. Université de Genève.

Hofstetter, R. \& Schneuwly, B. (2009). Introduction. Savoirs en (trans) formation. In R. Hofstetter \& B. Schneuwly (Eds.), Savoirs en (trans) formation. Au cour des professions de l'enseignement et de la formation, (p. 7-41). Bruxelles: De Boeck.

Johsua, S. (2002). Spécificités disciplinaires, spécificités didactiques: vers une didactique comparée. In P. Venturini, C. Amade-Escot, \& A. Terrisse. Études des pratiques effectives: l'approche des didactiques (p. 17-24). Grenoble: La Pensée sauvage.

Johsua, S. \& Dupin, J.-J. (1989). Représentations et modélisations: le "débat scientifique » dans la classe et l'apprentissage de la physique. Berne: Peter Lang.

Legrand, G. (1994). Les débats relatifs à un savoir scolaire: l'exemple de la grammaire. Spirale, 27-59.

Ligozat, F. (2008). Un point de vue de Didactique Comparée sur la classe de mathématiques. Étude de l'action conjointe du professeur et des élèves à propos de l'enseignement/apprentissage de la mesure de grandeurs dans des classes françaises et suisses romandes. Thèse de doctorat en sciences de l'éducation, FPSE, Université de Genève \& UFR Psychologie et sciences de l'éducation, Université de Provence.

Leutenegger, F. (2004). Le point de vue de la didactique comparée. Communication présentée au colloque de l'AIRDF, Québec, Août.

Magneron, N. \& Munier, V. (2008). Mesure et instrumentation dans l'enseignement scientifique. Aster 47.

Martinand, J.L. (1986). Connaître et transformer la matière. Berne: Peter Lang.

Martinand, J.L. (1996). Enseignement et apprentissage de la modélisation en sciences. Paris: Institut National de Recherche Pédagogique.

Mercier, A. Schubauer-Leoni, M.-L., \& Sensevy, G. (2002). Vers une didactique comparée. Revue française de pédagogie, 141, 5-16.

Monnier, A. \& Weiss, L. (2009). Des chiffres et des lettres. Tournez! Cahier de la Section des sciences de l'éducation, 121, Université de Genève.

Monnier, A., Weiss, L., de Marcellus, O., Erard, S., \& Staeger, P. (2008). Les observations vidéo peuvent-elles renforcer les liens entre théorie et pratique? Une expérience dans la formation initiale des maîtresses de l'enseignement secondaire genevois (IFMES). Revue des HEP de Suisse romande et du Tessin, 10, 175-193. 
Nonnon, E. (2000). Le tableau noir de l'enseignant, entre écrit et oral. Repères 22. 83-119.

Pastré, P. (2004). Introduction. Recherches en didactique professionnelle. In R. Samurçay \& P. Pastré. Recherches en didactique professionnelle (p. 1-14). Toulouse: Octares.

Perez, P. \& Rogalski, J. (2001). Interférences et conflits de schèmes dans l'usage d'outils professionnels: le cas d'un fichier cartographique de navigation. Le travail humain 2001/2, Volume 64, p. 145-172.

Prégent, R. (1990). La préparation d'un cours. Montréal: EEP.

Rabardel, P. (1995). Les hommes et les technologies. Une approche cognitive des instruments contemporains. Paris: Armand Colin.

Rey, B. (1998). Faire la classe à l'école élémentaire. Paris: ESF.

Riff, J. \& Durand, M. (1993). Planification et décision chez les enseignants. Bilan à partir des études en éducation physique et sportive, analyses et perspectives. Revue française de pédagogie, 103, 81-107.

Robardet, G. \& Guillaud, J.-C. (1997). Éléments de didactique des Sciences physiques. Paris: PUF.

Senore, D. \& Ubaldi, J.-L. (2006). Au cœur du quotidien. Six moments stratégiques pour faire la classe. In J.-L. Ubaldi. Débuter dans l'enseignement. Paris: ESF.

Schneuwly, B., Dolz, J., Aeby Daghé, S., Bain, D., Canelas-Trevisi, S., Cordeiro Sales, G., Gagnon, R., Jacquin, M., Ronveaux, Ch., Thévenaz-Christen, Th., Toulou, S. (2009). Des objets enseignés en classe de français. Le travail de l'enseignant sur la rédaction de textes argumentatifs et sur la subordonnée relative. Rouen: Presses universitaires de Rouen.

Schubauer-Leoni, M.-L. \& Leutenegger, F. (2002). Expliquer et comprendre dans une approche clinique/expérimentale du didactique « ordinaire ». In F. Leutenegger \& M. Saada-Robert (Ed.), Expliquer et comprendre en sciences de l'éducation (p. 227-251). Bruxelles: De Boeck.

Tricot, A., Plégat-Soutjis, F., Camps, J.-F., Amiel, A., Lutz, G., \& Morcillo, A. (2003). Utilité, utilisabilité, acceptabilité: interpréter les relations entre trois dimensions de l'évaluation des EIAH. Environnements informatiques pour l'apprentissage humain. Archives EIAH, 391-402.

Van der Maren, J.-M. (1999). La recherche appliquée en pédagogie. Des modèles pour l'enseignement. De Boeck Université, Bruxelles.

Wirthner, M. (2006). Le résumé d'un texte informatif s'enseigne-t-il? Des outils sémiotiques pour l'enseignant. In B. Schneuwly et Th. Thévenaz-Christen (Eds.). Analyses des objets enseignés. Le cas du français (p. 159-179). Bruxelles: De Boeck Université. 


\section{ANNEXE}

\begin{tabular}{|c|c|c|c|c|}
\hline Niveaux & $\begin{array}{l}\text { Repères } \\
\text { temporels }\end{array}$ & $\begin{array}{l}\text { Formes sociales } \\
\text { de travail }\end{array}$ & Matériel & Description \\
\hline 1 & $0-10$ min. & Collectif & & $\begin{array}{l}\text { Présentation de l'objectif de la leçon en lien } \\
\text { avec les leçons précédentes }\end{array}$ \\
\hline $1-1$ & $0-8 \min$. & $\begin{array}{l}\text { Démarche } \\
\text { question-réponse }\end{array}$ & & $\begin{array}{l}\text { Appel à la mémoire didactique de la } \\
\text { classe afin que les élèves se souviennent } \\
\text { des } 3 \text { caractéristiques principales de la } \\
\text { description: nommer les parties d'un tout, } \\
\text { les situer, les caractériser }\end{array}$ \\
\hline $1-2$ & $8-10 \mathrm{~min}$. & Travail d'écoute & & $\begin{array}{l}\text { Reformulation de l'objectif de la leçon sous } \\
\text { forme de problème: comment caractériser } \\
\text { les parties nommées? }\end{array}$ \\
\hline 2 & $10-55 \mathrm{~min}$ & & & $\begin{array}{l}\text { Observation des relatifs dans un texte } \\
\text { descriptif. }\end{array}$ \\
\hline $2-1$ & $10-15 \mathrm{~min}$. & Individuel & Texte & $\begin{array}{l}\text { Lecture à voix basse par les élèves du texte } \\
\text { distribué }\end{array}$ \\
\hline $2-2$ & $15-55 \mathrm{~min}$ & Groupes de 4 & Texte + questionnaire & Réponses à 4 questions \\
\hline $2-2-1$ & $15-25 \mathrm{~min}$ & Groupes de 4 & Texte + questionnaire & Souligner toutes les expansions du nom \\
\hline $2-2-2$ & $25-35 \mathrm{~min}$. & Groupes de 4 & Texte + questionnaire & $\begin{array}{l}\text { Classer les expansions du nom soulignées } \\
\text { dans un tableau personnel }\end{array}$ \\
\hline $2-2-3$ & $35-45 \min$ & Groupes de 4 & Texte + questionnaire & $\begin{array}{l}\text { Vérifier dans quelle mesure on peut } \\
\text { substituer une expansion par une autre }\end{array}$ \\
\hline $2-2-4$ & $45-55 \mathrm{~min}$ & Groupes de 4 & Texte + questionnaire & $\begin{array}{l}\text { Vérifier dans quel cas on ne peut pas } \\
\text { supprimer la relative }\end{array}$ \\
\hline
\end{tabular}

